\title{
A Comparison of the Diabetes-related Attitudes of Health Care Professionals and Patients
}

\author{
Robert M. Anderson, EdD ${ }^{\mathrm{a}}$, James T. Fitzgerald, $\mathrm{PhD}^{\mathrm{a}}$, Daniel W. Gorenflo, $\mathrm{PhD}^{\mathrm{b}}$ \\ and Mary S. Oh, BS \\ ${ }^{a}$ University of Michigan Medical School, Michigan Diabetes Research and Training Center, Department of Postgraduate \\ Medicine/Health Professions Education, Ann Arbor, Michigan 48109-0201 and ${ }^{b}$ The University of Michigan Medical School, Depart- \\ ment of Family Practice, 1018 Fuller, Box 0708, Ann Arbor, Michigan 48109-0708 (USA)
}

(Received June 12th, 1992)

(Accepted November 3rd, 1992)

\begin{abstract}
The following study compares the diabetesrelated attitudes of sampled health care professionals and patients with diabetes. Attitudes were measured with a revised version of the Diabetes Attitude Scale (DAS) which includes seven factors representing attitudes towards: (1) the need for special training in order to provide diabetes care; (2) patient compliance; (3) the seriousness of noninsulin-dependent diabetes (NIDDM); (4) the relationship between blood glucose levels and the complications of diabetes; (5) the impact of diabetes on the patient's life; (6) patient autonomy; and (7) team care. The highest levels of agreement among patients and professionals concerned the seriousness of NIDDM and the relationship between blood glucose control and the development of the complications of diabetes. The most striking finding of the study was that patients tended to express a significantly more judgmental, moralistic attitude toward patient behavior than did health care professionals.
\end{abstract}

Key words: Attitudes; Patients; Professionals; Diabetes.

\section{Introduction}

Diabetes is a lifelong chronic disease in which patients deliver over $95 \%$ of their own care. This fact makes patient education the crucial component of good diabetes care. Patients must acquire the technical skills required for diabetes management in order to achieve a complex balance among medications, diet, exercise and lifestyle. Some of the major theories of health behavior and health education, such as the health belief model [1] and the theory of reasoned action [2], emphasize that attitudes and beliefs are a major component of health behavior. Understanding the behavior of persons with diabetes and responding to their needs with an appropriate program of patient education requires some knowledge of their attitudes towards diabetes and its care [3].

Attitudes also influence the behavior of health care professionals $[2,4]$. Since patient education is largely a process of communication between health care professionals and their patients it is important to understand the similarities and differences in their attitudes towards diabetes and its treatment. The purpose of this paper is to compare the diabetesrelated attitudes of physicians, nurses and dietitians with those of diabetic patients and to discuss the implications of those comparisons for diabetes patient education. The diabetes-related attitudes of patients and 
health care professionals reported in this paper were measured with a revised version of the Diabetes Attitudes Scale (DAS) [5].

The content of the original DAS was developed by a national panel of 17 diabetes experts to measure the attitudes of health care professionals. The panel included three physicians, three nurses, four nutritionists, three consumers of diabetes care and four behavioral scientists. The group interacted by mail through a modified Delphi [6] process. Members were asked to write Likert type attitude items, i.e. statements that elicit reaction on a five-point scale indicating a respondent's degree of agreement or disagreement with the statements. To ensure the comprehensiveness of the DAS, the panel was asked to write items covering four global areas of diabetes: the disease itself, the treatment of diabetes, diabetic patients and professional education in diabetes. The panel wrote a total of 347 attitude items: 62 items related to the disease itself, 135 items related to treating diabetes, 92 items focusing on the individuals with diabetes, and 58 items concerned with diabetes professional education.

The panel members reviewed all 347 items, suggested wording changes, indicated whether the correct response to an item was to agree or disagree and selected 20 items from each of the four areas that they believed addressed important issues in diabetes and should therefore be included in the final scale. The group was asked to indicate the correct response for each item to provide a criterion for desirable or appropriate attitudes. This criterion was established because one of the intended uses of the scale was the evaluation of professional education programs. Items that had at least an $80 \%$ level of agreement on the direction of the appropriate response and more than five votes for inclusion were included in a preliminary version of the scale. This resulted in an instrument for which there was a high level of agreement among the panel of diabetes experts regarding both the significance of the items and their correct responses.
The preliminary version of the scale, which contained 60 items, was pilot-tested using a convenience sample of 60 health care professionals. An item analysis, examining item variability and inter-item correlations, was performed. This analysis led to the elimination of 10 items.

Psychometric analyses of the 50 items, using a sample of 633 nurses, 322 dietitians and 116 physicians, identified eight factors. These factors represented professionals' attitudes toward: (1) the need for special training in the treatment of diabetes; (2) the importance of blood glucose control in minimizing the complications of diabetes; (3) the role of the patient in diabetes self-care and management; (4) patients' commitment to controlling their disease; (5) the importance of a team approach to diabetes care; (6) the seriousness of noninsulin-dependent diabetes (NIDDM); (7) the difficulties in treating diabetes; and (8) the efficacy of outpatient education. Information about the reliability and validity of the DAS has been published elsewhere [7].

During the review of the responses to the DAS by physicians, nurses and dietitians, it became clear that it would be useful to know how persons with diabetes viewed these same issues. Additionally, a DAS that could be completed by both patients and health care professionals would allow for direct comparisons of the diabetes-related attitudes of both groups. During the assessment of the potential of the DAS to measure the attitudes of patients, it was decided that the instrument would need to be changed to make its wording less technical. The authors rewrote most of the items to make them less technical while trying to retain their original meaning. A study [8] was carried out in which two random samples of health care professionals were sent either the original DAS or the revised DAS. A comparison of the responses of health care professionals to the original DAS and the revised DAS clearly indicated that the revisions had changed the psychometric properties of the attitude scale. Based on these 
results it was determined that the revised DAS would have to be viewed as a new attitude measure and its psychometric properties would have to be established based on administrations of the scale to patients [9].

The revised DAS can be used to assess the impact of diabetes education programs on the attitudes of both patients and professionals as well as to explore the relationship between the attitudes and behavior of both groups. The revised DAS also represents a general measure of patients' attitudes toward diabetes. As such, it can be used to compare the attitudes of patients and health care professionals. This comparison can provide an opportunity to identify differences in opinion that could influence the quality of patientprofessional communication and thereby affect the management of the disease.

\section{Materials and Methods}

\section{The revised Diabetes Attitude Scale}

Like the original DAS, the revised DAS consists of 50 Likert scale items $(5=$ strongly agree; $4=$ agree; $3=$ neither agree or disagree; $2=$ disagree and $1=$ strongly disagree). Forty-one of the original 50 items were rewritten to eliminate technical terms; nine others were left unchanged. The content of the items on the revised DAS closely matches that of the original items; however, as previously noted, psychometric testing has determined that the meanings of some of the items were changed as a result of the modification process. Although the revisions were not specifically intended to lower the reading level of the instrument, the revised DAS is written at the 10th grade level, as opposed to the 12th grade level of the original.

\section{Study participants}

Surveys incorporating the revised DAS were mailed to two samples of diabetic patients. The first sample consisted of 1054 patients who had attended the University of Michigan Hospitals' diabetes clinic. The survey was returned by 419 patients in this sample for a return rate of $40 \%$. To broaden the sample of patients, the survey was also sent to 1003 patients living in nine Michigan communities and receiving a monthly diabetes newsletter. Patients in this sample returned 823 surveys for a return rate of $82 \%$, giving an overall return rate of $60 \%$ (1242 of 2057). Forty surveys were subsequently eliminated from the analyses because the patients did not meet the age criterion of 16 years or older, so the final analysis sample consisted of 1202 patients.

The revised DAS was also mailed to 500 nurses, 500 registered dietitians and 1000 primary care physicians, as part of a survey designed for health care professionals. Physicians were oversampled because of the documented difficulty [10] in getting physicians to complete mailed surveys. Since the great majority of persons with diabetes receive their care from primary care practitioners, efforts were made to ensure that this mailing would go to physicians, nurses and dietitians who did not specialize in the treatment of diabetes. Surveys were returned by 149 physicians, 227 registered dietitians and 189 registered nurses for a total of 573 surveys completed by health care professionals. The return rate was $15 \%$ for physicians, $45 \%$ for dietitians and $38 \%$ for nurses. The following study is based on an analysis of 1744 diabetes attitude surveys from both patients and health professionals. (Table 1).

\section{Statistical methods}

A confirmatory factor analysis using patients and health care professionals provided a seven factor solution very similar to that found in an earlier DAS study using only patients [9]. The internal consistency of the seven DAS subscales was then determined through the use of Cronbach's alpha (Table 2). To determine whether attitudes on the seven DAS scales differed between six groups (physicians, dietitians, nurses, patients with insulin-dependent diabetes (IDDM), patients with noninsulin-dependent diabetes (NIDDM) who do not use insulin and NIDDM pa- 
Table 1. Composition of Sample by Health Care Profession and Type of Diabetes $(n=1744)$.

\begin{tabular}{lll}
\hline Group & Number & $\begin{array}{l}\text { Percent of } \\
\text { sample }\end{array}$ \\
\hline Physicians & 149 & 8.5 \\
Registered Dietitians & 227 & 13.0 \\
Registered Nurses & 189 & 10.8 \\
IDDM patients & 410 & 23.5 \\
NIDDM patients & 445 & 25.5 \\
$\quad$ using insulin & & 18.6 \\
$\quad$ NIDDM patients & 324 & \\
\hline
\end{tabular}

tients who do use insulin) a multivariate analysis of variance (MANOVA) was performed. A Scheffé post hoc test was used to determine specific group differences for each attitude subscale.

\section{Results}

Overall differences in the DAS scores

To test for overall attitudinal differences among physicians, dietitians, nurses, IDDM patients, NIDDM patients using insulin and NIDDM patients not using insulin, a MANOVA was performed on the seven DAS subscales. The results indicated a signifi-
Table 3. MANOVA test results.

\begin{tabular}{|c|c|c|c|c|}
\hline & & $\begin{array}{l}\text { Mean } \\
\text { Square }\end{array}$ & $F$-value & $P>F$ \\
\hline 1. & $\begin{array}{l}\text { Special } \\
\text { training }\end{array}$ & 4.47 & 28.64 & 0.0001 \\
\hline 2. & $\begin{array}{l}\text { Patient } \\
\text { compliance }\end{array}$ & 47.24 & 141.63 & 0.0001 \\
\hline 3. & $\begin{array}{l}\text { Seriousness } \\
\text { of NIDDM }\end{array}$ & 1.92 & 3.23 & 0.0065 \\
\hline 4. & $\begin{array}{l}\text { Control/com- } \\
\text { plications }\end{array}$ & 0.69 & 2.55 & 0.0262 \\
\hline 5. & $\begin{array}{l}\text { Impact of } \\
\text { diabetes }\end{array}$ & 3.61 & 11.38 & 0.0001 \\
\hline 6. & $\begin{array}{l}\text { Patient } \\
\text { autonomy }\end{array}$ & 10.43 & 27.02 & 0.0001 \\
\hline 7. & Team care & 4.97 & 20.54 & 0.0001 \\
\hline
\end{tabular}

cant main effect of group (Wilks' Lambda $P=0.0001$ ). In addition, univariate $F$-tests showed a significant effect for group on all seven subscales (Table 3 ). Table 4 provides the subscale means for each group. Specific group differences for each subscale are discussed below.

DAS Subscale 1. Special Training (The attitude that health care professionals need special training to care for persons with diabetes)

The Scheffé test indicated that physicians,

Table 2. Descriptive statistics for subscales.

\begin{tabular}{llllll}
\hline Subscale & $N^{\mathrm{a}}$ & Mean & S.D. & Range & $\begin{array}{l}\text { Cronbach's } \\
\text { alpha }\end{array}$ \\
\hline 1. Special training & 1695 & 4.25 & 0.41 & $2.43-5.00$ & 0.72 \\
2. Patient compliance & 1697 & 3.29 & 0.69 & $1.17-5.00$ & 0.72 \\
3. Seriousness of NIDDM & 1712 & 3.50 & 0.77 & $1.00-5.00$ & 0.59 \\
4. Control/complications & 1709 & 4.24 & 0.52 & $1.75-5.00$ & 0.66 \\
5. Impact of diabetes & 1695 & 4.09 & 0.57 & $1.80-5.00$ & 0.64 \\
6. Patient autonomy & 1699 & 3.66 & 0.64 & $1.00-5.00$ & 0.62 \\
7. Team care & 1712 & 4.19 & 0.51 & $1.00-5.00$ & 0.68 \\
\hline
\end{tabular}

${ }^{\text {a The }} N$ varies because not every respondent answered every item. 
Table 4. Subscale means for each health care professional group and patient group.

\begin{tabular}{|c|c|c|c|c|c|c|c|}
\hline \multirow[t]{2}{*}{ Group } & \multicolumn{7}{|c|}{ Subscale } \\
\hline & 1 & 2 & 3 & 4 & 5 & 6 & 7 \\
\hline Physicians & 3.95 & 2.88 & 3.60 & 4.16 & 4.00 & 3.58 & 3.97 \\
\hline Registered dietitians & 4.41 & 2.75 & 3.62 & 4.25 & 4.04 & 3.92 & 4.46 \\
\hline Registered nurses & 4.13 & 2.77 & 3.60 & 4.24 & 4.09 & 3.79 & 4.22 \\
\hline IDDM patients & 4.28 & 3.26 & 3.46 & 4.28 & 4.25 & 3.82 & 4.13 \\
\hline $\begin{array}{l}\text { NIDDM patients } \\
\text { using insulin }\end{array}$ & 4.31 & 3.67 & 3.49 & 4.27 & 4.10 & 3.50 & 4.19 \\
\hline $\begin{array}{l}\text { NIDDM patients } \\
\text { not using insulin }\end{array}$ & 4.23 & 3.68 & 3.40 & 4.17 & 3.94 & 3.45 & 4.15 \\
\hline
\end{tabular}

who had the lowest score (3.95) for this subscale, differed from every other group. Dietitians had the highest score (4.41) and they differed from all other groups with the exception of NIDDM patients using insulin. Nurse's scores were closer to the physicians (4.13) and differed with all groups except NIDDM patients not using insulin. Patients' mean scores were higher than physicians' and nurses' scores. The three patient groups did not differ significantly. IDDM patients differed from each group of health professionals.

DAS Subscale 2. Patient Compliance (The attitude that patients should do what they are told to do by health care professionals. A number of the items on this subscale have a moralistic, blaming tone)

A distinct division between health care professionals and patients is evident for this subscale, with patients agreeing much more strongly with the items in this subscalc. Health professionals had lower scores (M.D. $=2.88$, R.D. $=2.75$ and R.N. $=2.77$ ) that were not significantly different from each other. NIDDM patients had the highest scores (Using insulin $=3.67$ and Not using insulin $=3.68$, while IDDM patients had an intermediate score (3.26). Bach of the health professional groups differed from each of the patient types. Both NIDDM patient groups differed from IDDM patients. NIDDM patients agreed that patients should follow the instructions of their health care provider, while IDDM patients were more neutral. Interestingly, the health care professionals were generally neutral on this attitude scale.

DAS Subscale 3. Seriousness of NIDDM (The attitude that NIDDM is a serious disease)

Health professionals had the higher scores, but the means test did not reveal any significant differences among any groups. Overall, all groups of professionals and patients viewed NIDDM as a serious condition, but not at a very strong level of agreement with the statement

DAS Subscale 4. Control/Complications (The perception of a relationship between high blood glucose levels and the development of the complications of diabetes)

The means test did not reveal any significant differences among the groups. The professionals and patients agreed that there is a relationship between level of blood glucose control and the development of the complications of diabetes.

DAS Subscale 5. Impact of Diabetes ( The attitude that diabetes has a significant negative impact on the patient's life)

IDDM patients scored the highest for this 
subscale (4.25) and differed from all groups except nurses. NIDDM patients using insulin (4.10) differed with NIDDM patients not using insulin (3.94). The three health professional group's scores did not differ significantly. As a whole, all groups agreed that diabetes had a negative impact on patient lives.

DAS Subscale 6. Patient Autonomy (The attitude that the patient should be the primary decision maker regarding the daily self-care of diabetes)

For this subscale, nurses (3.79), dietitians (3.92) and IDDM patients (3.82) had the highest scores. NIDDM patients not using insulin (3.45), NIDDM patients using insulin (3.50) and physicians (3.58) had the lowest scores. NIDDM patients differed from nurses, dietitians and IDDM patients. IDDM patients also differed from physicians. Within the health professionals, physicians differed from dietitians. Although all groups agreed the patient should be the primary decision maker in the daily treatment of diabetes, dietitians, IDDM patients and nurses were more supportive of this attitude than were NIDDM patients and physicians.

\section{DAS Subscale 7. Team Care (The attitude that} nurses and dietitians are needed in the care of diabetes.)

The scores on this subscale are similar to the scores on Special Training subscale in that dietitians had the highest score (4.46) and physicians the lowest (3.97), with the dietitians' score differing from all other groups. The physicians' score differed from all except IDDM patients. The three patients' scores did not significantly differ. While dietitians agreed most strongly to a team approach to diabetes care, all groups agreed to the importance of a team care approach.

\section{Discussion}

\section{Differences within subscale by patient and pro- fessional group \\ To clarify the findings of the study for the}

purposes of discussion, groups of patients and/or professionals whose attitudes were not significantly different statistically have been grouped together for the discussion of each subscale.

DAS Subscale 1 - Special Training. All of the groups agreed with the need for health care professionals to have special training in order to treat diabetes. However, dietitians agreed most strongly (4.41), followed by patients (4.28), nurses (4.13) and finally physicians (3.95). It is interesting to note that physicians were the least supportive while dietitians were the most. The physician respondents to this survey were for the most part primary care physicians who treat a wide variety of conditions. They are by training and practice, generalists and it is therefore not surprising that they expressed the least support for the need for special training. Nutritionists on the other hand, require special expertise to provide care for diabetic patients, because diabetes is a disease involving altered metabolism. In addition to knowledge of basic nutrition and metabolism, dietitians are required to understand the altered metabolism and concomitant special nutritional requirements of diabetic patients. As for the other groups, nurses and patients generally expressed fairly strong support for the need for special training.

DAS Subscale 2-Patient Compliance. The findings on this subscale are the most striking in this study. Subscale 2 has a strong moralizing tone, it includes statements such as 'people who do not follow their recommended diabetes treatment don't really care about controlling their diabetes,' and 'controlling their diabetes should be the most important thing in the lives of people with diabetes.' NIDDM patients expressed the strongest agreement (3.68) with this subscale, followed by IDDM patients (3.26) and then by health professionals (2.79). These findings indicate that patients were much more likely than health professionals to express agreement with statements written in a judgmental or even blaming tone. In fact, the mean scores of 
the health care professionals were below the scale midpoint (3.00), indicating that health care professionals were much more likely than patients to disagree with statements of this type.

DAS Subscale 3 - The Seriousness of NIDDM. There was no significant difference between any of the groups on this subscale, indicating that health care professionals and patients were similar in their agreement that NIDDM is a serious disease. However, it should be noted that the strength of their belief that NIDDM was a serious disease was relatively weak $(3.50$ - see Table 2$)$.

DAS Subscale 4 - Control/Complications: Again, there were no differences among any of the groups in their attitudes toward the relationship between blood glucose control and the development of the complications of diabetes. All of the groups expressed strong support for the concept that hyperglycemia contributes to the development of complications.

DAS Subscale 5 - The Impact of Diabetes IDDM patients expressed the strongest agreement (4.26) with the idea that diabetes has a negative impact on a patient's life. NIDDM patients who use insulin also agreed strongly with the subscale (4.10), followed by health professionals (4.05), and NIDDM patients who do not use insulin (3.95). The differences among patients support the construct validity of the scale. IDDM patients who have a more serious form of diabetes would be expected to express the strongest agreement about the negative impact of the disease. The next strongest level of agreement was expressed by NIDDM patients who use insulin. In previous studies [12,13] as well as in the present investigation, we have classified NIDDM patients as to whether they use insulin, based on the differing perceptions of disease severity of insulin use or non-use. The use of insulin conveys to paticnts a message about the seriousness of the disease.

DAS Subscale 6 - Patient Autonomy. IDDM patients, nurses and dietitians express- ed the strongest agreement (3.84) with the belief that patients should make their own decisions about the daily treatment of diabetes. Physicians and NIDDM patients were significantly weaker (3.49) in their support for the concept of patient autonomy. These results are consistent with differences in the various roles of these health professionals and patients. The most common role of both nurses and dietitians is that of diabetes educator. In such capacity, they are committed to helping patients make the necessary preparation to enable themselves to manage diabetes self-care and to participate in its planning. Conversely, physicians are trained by the health care system to be responsible for treatment decisions and to make these decisions unilaterally, so it is not surprising that they tend to express less support for the concept of patient autonomy. IDDM patients are required to make frequent and often complex decisions about the management of their own disease for most of their lives. NIDDM patients tend to be older than IDDM patients and older diabetic patients are more likely to prefer to have the physician be the primary decision-maker regarding diabetes care [9].

DAS Subscale 7 - Team Care. Dietitians were strongest in their agreement (4.46) with the importance of dietitians and nurses in diabetes care, followed by nurses and patients (4.17) and finally by physicians (3.97). Dietitians undergo a significant amount of training to prepare them to provide the nutritional treatment of diabetes. They are often trained to function as spccialists and are likely to operate as part of a diabetes care team. On the other hand most of the primary care physicians in this study were trained and function as generalists. Furthermore, in many community settings physicians are the sole provider of diabetes care, such training and experience help explain their weaker support for this concept.

Table 5 represents our judgement about the implications of these findings for the practice of diabetes patient education. 
Table 5. Implications for practice. Factors and suggestions for their clinical application.

\section{Attitude towards the need for special training}

The most important discrepancy in this factor is between the attitudes of primary care physicians and patients with diabetes. All groups of patients expressed strong support for the need for special training for health care professionals. This issue will become more important as the treatment of diabetes becomes more complex, through the increasing use of sophisticated insulin delivery systems and blood glucose monitoring technologies. Because the number of endocrinologists is so small in comparison to the number patients with diabetes, it is likely that the specialized role of the diabetes patient educator will become more important. Diabetes educators possess a body of specialized knowledge, encompassing technical, psychological and educational facets; patients and primary care practitioners will become increasingly more reliant upon this knowledge. The development of a certification exam [14] for diabetes educators and the publication of the scope of practice and of the standards of practice for diabetes educators [15] attest to the increasing recognition of diabetes patient education as a genuine medical specialty.

\section{Attitudes toward patient compliance}

The striking finding that patients were much more judgmental about patient behavior than health care professionals suggests to us an important clue in understanding the way current educational approaches may be perceived by patients. It appears that they hear in these current approaches a definite general criticism of patient compliance. We think that criticizing patients is likely to be an ineffective educational and behavior modifying technique. We believe that criticism is likely to increase patients' feelings of guilt and self-blame, thus adding to the psychological burden of having diabetes, without necessarily producing any measurable increase in responsibility or adherence. We believe that a nonjudgmental, collaborative, problem-solving approach would be more productive.

3. Attitude towards the seriousness of noninsulin-dependent diabetes (NIDDM)

Although patients and health care professionals are similar in their level of agreement about the seriousness of NIDDM, that agreement is not very strong. This finding has important implications for patient education because NIDDM require difficult and long-lasting behavior changes on the part of patients. It is unlikely that such changes will be made and sustained unless patients and health care professionals alike understand and accept the serious nature of NIDDM.

4. Attitude towards the relationship of blood glucose levels and complications

The fact that most health care professionals and patients believe in the relationship between blood glucose control and the complications of diabetes can be viewed as a positive finding. However, educators must be careful not to implicitly or explicitly guarantee to patients that they will escape the complications of diabetes if they engage in an aggressive effort to control their blood glucose. It is also important for educators to help patients minimize guilt and self-blame if they do develop the complications of diabetes.

5. Attitude towards the negative impact of diabetes on the patient's life

This study suggests that health care professionals tend to underestimate the perceived negative impact that diabetes has on the lives of patients who are required to take insulin. In some of the Michigan Diabetes Research and Training Center's professional education programs we require that health care professionals follow three to five days of simulated diabetes self-care, including injections of mock insulin (using a saline solution). We have been surprised by the number of diabetes educators, who have been teaching patients selfinjection for years, who, when confronted with the same task themselves, are either unwilling to give themselves injections or find it extremely difficult and upsetting. This finding suggests that diabetes educators need to be sure they are aware of the emotional impact that taking insulin has for patients. For many patients taking insulin is traumatic; not only do they dislike giving themselves the injections but the need to take insulin conveys to them the message that diabetes is a much more serious disease than they had previously believed. 
6. Attitude towards patient autonomy

Physicians and NIDDM patients were weakest in their support for patient autonomy while IDDM patients, nurses, and dietitians were the strongest. Diabetes care, especially when it involves the adininistration of insulin, requires that the patient be able to make complex daily treatment decisions. Having the patient make treatment decisions may be counter-intuitive for physicians who have been trained to make such decisions themselves. Diabetes self-care often requires a high degree of patient autonomy. Diabetes educators may have an important role to play in facilitating the delegation of responsibility for making treatment decisions from physicians to patients.

\section{Attitude towards team care}

Dietitians and patients view diabetes as a complex disease requiring a multidisciplinary team approach to care; they hold this view more strongly than primary care physicians do. This finding suggests that it is important for physicians to understand the benefits of team care, because it can not thrive without their active participation and support.

\section{Study limitations}

The major limitation of this study is the low return rate for health care professionals, especially physicians. Although it was possible to increase the absolute number of physicians studied by oversampling, this procedure does not usually increase a low return rate, which limits the generalizability of these findings to the larger population of health care professionals. In order to determine the seriousness of this problem, the scores of these health care professionals were compared to earlier diabetes attitudes studies involving health care professionals with higher return rates $[7,11]$. The comparison showed that scores on the revised DAS tended to be consistently more favorable than the original DAS across all subscales and professional groups. However, the relationships of the subscale scores among health care professionals was consistent for both instruments whether or not the sample was diabetes specialists or primary care generalists. For example, on a given subscalc where physicians have the lowest scores and dietitians have the highest score, that relationship remained consistent no matter which version of the instrument was used or whether the respondents were diabetes specialists or general practitioners. This finding indicates that the revised
DAS provides a fair representation of differences among the various health care professional and patient groups. Since the major purpose of this study was a comparison of attitudes across groups, we feel that the findings are valid and useful.

\section{Acknowledgments}

This study was supported in part by a grant from the National Institutes of Health, \#5P608DK20572, National Institute of Diabetes, Digestive and Kidney Diseases.

\section{References}

1 Becker MH, Janz NK: The health belief model applied to understanding diabetes regimen compliance. Diabetes Educ 1985; 11: 41-47.

2 Ajzen I, Fishbein M: Understanding attitudes and predicting social behavior. Englewood Cliffs, NJ: Prentiss Hall, 1980 .

3 Anderson RM, Nowacek GW, Richards F: Influencing the personal meaning of diabetes: Research and practice. Diabetes Educ 1988; 14: 297-302.

4 Weinberger M, Cohen SJ, Mazzuca SA: The role of physicians knowledge and attitudes in effective diabetes management. Soc Sci Med 1984; 19: 965-969.

5 Anderson RM, Gressard C: Developing a measure of the attitudes of health care providers towards diabetes and its treatment. Diabetes 1987; 36: 120A

6 Udinsky BF, Ostorlind SJ, Lynch S: DELPHI technique. 
In: Evaluation resource handbook: gathering, analyzing, reporting data. San Diego, CA: edits, 1981.

7 Anderson RM, Donnelly MB, Grcssard C, Dedrick RF: The development of a diabetes attitude scale for health care professionals. Diabetes Care 1989; 12: 120-127.

8 Anderson RM, Donnelly MB: Words and meanings: a cautionary tale for diabetes educators. Diabetes Educ 1990; 16: 117-122.

9 Anderson AM, Donnelly MB, Dedrick RF: Measuring the attitudes of patients towards diabetes and its treatment. Patient Educ Couns 1990; 16: 231-245.

10 Maleux B, Legault C, Lambert J: Increasing response rate in physicians' mail surveys: An experimental study. Am J Public Health 1989; 638-639.

11 Anderson RM, Donnelly MB, Dedrick RF and Gressard CP: The attitudes of nurses, dietitians and physicians toward diabetes. Diabetes Educ 1991; 17: 261-268.

12 Anderson AM, Hess GE, Davis WK, Kiss RG: Communi- ty diabetes care in the 1980s. Diabetes Care 1988; 11 : 519-526.

13 Anderson RM, Lockwood D, Dedrick RF, Hiss RG: The diabetes care and education provided by nurses working in physicians' offices. Diabetes Educ 1988; 14: 532-536.

14 Steil, C, Anderson B: CDE's in 1990: A report of years 3 and 4 (1988 and 1989) of the certification process. Diabetes Educ 1991; 17: 20-22.

15 Task Force of the American Association of Diabetes Educators: The scope of practice for diabetes educators and the standards of practice for diabetes educators. Diabetes Educ 1992; 18: 52-56.

Correspondence to:

Robert M. Anderson, EdD

The University of Michigan Medical School

The Towsley Center, Room 1116, Box 0201

AM Arbor, Michigan 48109-0201, USA. 